\title{
The multiple roles and therapeutic potential of clusterin in non-small-cell lung cancer: a narrative review
}

\author{
Juofang Tan", Wei Guo", Su Yang, Dingpei Han, Hecheng Li \\ Department of Thoracic Surgery, Ruijin Hospital, Shanghai Jiao Tong University School of Medicine, Shanghai, China \\ Contributions: (I) Conception and design: J Tan, W Guo, H Li; (II) Administrative support: H Li; (III) Provision of study materials or patients: None; \\ (IV) Collection and assembly of data: All authors; (V) Data analysis and interpretation: All authors; (VI) Manuscript writing: All authors; (VII) Final \\ approval of manuscript: All authors. \\ \#These authors contributed equally to this work. \\ Correspondence to: Hecheng Li. Department of Thoracic Surgery, Ruijin Hospital, Shanghai Jiao Tong University School of Medicine, Shanghai \\ 200025, China. Email: lihecheng2000@hotmail.com.
}

\begin{abstract}
Worldwide, lung cancer is the most common form of cancer, with an estimated 2.09 million new cases and 1.76 million of death cause in 2018. It is categorized into two subtypes, small-cell lung cancer (SCLC) and non-small-cell lung cancer (NSCLC). Although platinum-based chemotherapy or molecular targeted drugs is recommended for advanced stages of NSCLC patients, however, resistance to drug and chemotherapy are hindrances for patients to fully beneficial from these treatments. Clusterin (CLU), also known as apolipoprotein $\mathrm{J}$, is a versatile chaperone molecule which produced by a wide array of tissues and found in most biologic fluids. There are studies reported high expression of CLU confers resistance to chemotherapy and radiotherapy in different lung cancer cell lines. By silencing CLU using Custirsen (OGX011), a second-generation antisense oligonucleotide (ASO) that inhibits CLU production, not only could sensitized cells to chemo- and radiotherapy, also could decreased their metastatic potential. We will review here the extensive literature linking CLU to NSCLC, update the current state of research on CLU for better understanding of this unique protein and the development of more effective anti- CLU treatment.
\end{abstract}

Keywords: Non-small-cell lung cancer (NSCLC); clusterin (CLU); chemotherapy; radiotherapy; antisense oligonucleotide

Submitted Dec 25, 2020. Accepted for publication Apr 19, 2021.

doi: $10.21037 /$ tlcr-20-1298

View this article at: http://dx.doi.org/10.21037/tlcr-20-1298

\section{Introduction}

Lung cancer is the leading cause of cancer-related deaths worldwide, incidence and mortality rates of lung cancer have been ballooning in recent years. The etiologic factors of lung cancer have become more complex along with environmental pollution, urbanization, and industrialization problems around the world. In 2018, it is estimated that 2.09 million new cases of lung cancer $(11.6 \%$ of total cancer cases) and 1.76 million of death cause by lung cancer (18.4\% of total cancer death) occurred all over the world, ranking first in the most frequent cancer and cause of cancer death among all cancer types, in combined of men and women (1).
The American Cancer Society's estimates that fresh cases of lung cancer in the United States for 2020 are about 228,820 (116,300 in men and 112,520 in women) and 135,720 deaths from lung cancer in 2020 (72,500 in men and 63,220 in women) (2).

Histologically, lung cancer could be categorized into two subtypes, small-cell lung cancer (SCLC) and non-small-cell lung cancer (NSCLC). The World Health Organization (WHO) has classified NSCLC into adenocarcinoma, squamous cell carcinoma and large cell carcinoma (3). Surgery is still recommended for patients with early stages of NSCLC, while platinum-based chemotherapy or molecular targeted drugs remains the first-line treatment 
for advanced stages (4). However, the 5-year survival rate is still considered low (<7\%) (5). SCLC, accounts for approximately $14 \%$ of all lung cancers, highly metastatic and rapid growth contribute SCLC to a high mortality rate and low survival rate, most patients survive for only a year or less (6). Thus, it is rarely possible for surgical resection, chemotherapy and/or radiotherapy became the remaining options. Although the combination of radiotherapy with either surgery or chemotherapy treatment proven could improve the survival of SCLC patients $(7,8)$, still, the general 5 -year survival rate of people with SCLC patients is $6 \%(5)$.

Clusterin (CLU), also known as apolipoprotein $\mathrm{J}$, testosterone-repressed prostate message-2 (TPRM-2), sulphated glycoprotein-2 (SGP-2) and compliment lysis inhibitor (CLI), was first isolated from ram rete testes fluid in 1986, they showed that a heat-stable, trypsin sensitive protein was responsible to aggregate cells, so they named this extracellular cell 'Clusterin' (9). CLU is a highly conserved glycoprotein found nearly ubiquitous in tissues and body fluids (10). In human, CLU was described as CLI in 1989 firstly, a component of soluble terminal complement complexes immunologically identified in human seminal plasma, playing an important role in protecting sperm cells and epithelial tissues against complement attack in the male reproductive system (11). Since then, CLU has been found implicated in many processes, which included apoptosis, cell cycle regulation and DNA repair (12-16).

Several studies have reported high expression of CLU in different lung cancer cell lines $(17,18)$. Compared to normal lung cancer cells, CLU expression was found higher in drug-resistant lung cancer cell lines, which indicates that CLU might be involved in drug resistant. Moreover, CLU could also represent an independent prognostic factor in surgically resected lung cancer patients $(19,20)$.

In this review, we will firstly discuss the structure and physiology function of CLU, then about the role of CLU in tumorigenesis, metastasis, chemotherapy and radiotherapy in lung cancer.

The information used to write this review was collected from PUBMED database (date of the last search 14 March 2021), using combinations of search terms including "lung cancer", "clusterin", "apolipoprotein J", "chemotherapy", "radiotherapy" and "antisense oligonucleotide". Reference lists of identified articles were searched manually to find other relevant studies. We present the following article in accordance with the Narrative Review reporting checklist (available at http://dx.doi.org/10.21037/tlcr-20-1298).

\section{Structure and biological function of CLU}

\section{Structure of $C L U$}

Human CLUI predominant form is a secreted heterodimeric glycoprotein, containing about $30 \%$ of $\mathrm{N}$-linked carbohydrate rich in sialic acid. It is located at chromosome 8 p21-p12 and is organized in 8 introns and 9 exons of different size, resulting in a gene with a total length of $17,876 \mathrm{bp}$ (base pair) and is comprised of two disulfidelinked subunits designated CLU $1(34-36 \mathrm{kDa})$ and CLU $2(36-39 \mathrm{kDa})$, each containing three cysteines involved in disulfide bonds. The $\mathrm{N}$-linked carbohydrate is the site of sulfation, with heterogeneity of glycosylation in different sites. When chemically deglycosylated, the subunits have molecular masses of 24 and $28 \mathrm{kDa}$, respectively.

Several mRNAs isoforms have been transcribed from the alternative use of $C L U$ gene exon 1, it involved into three different following portions: $1 \mathrm{a}, 1 \mathrm{~b}$ and $1 \mathrm{c}$, and they share the remaining exon 2 to $9(21,22)$. The most extensively studied of the human CLU is the secretory CLU (sCLU), a $75-80 \mathrm{kDa}$ heterodimer present in almost all physiological fluids. Another isoform is the nuclear clusterin (nCLU), a $55 \mathrm{kDa}$ protein found inside the nucleus of the cell. The third isoform which remain poorly unknown, was found mostly in the cytoplasm, is the cytoplasmic clusterin (cCLU) $(23,24)$.

\section{Molecular function of $C L U$}

Different studies confirmed this dichotomous role of CLU isoforms related to apoptosis. A possible link with apoptotic death was found a long time ago (25). Studies have been carried out investigating on this issue. Through exposing cancer cells to ionizing radiation (IR), the C-terminal coiled-coil domain of nCLU formed a complex with $\mathrm{Ku} 70 /$ $\mathrm{Ku} 80$, resulting in reduction of cell growth and colonyforming ability, concurrent with increased G1 cell cycle arrest and cell death (26). Another study revealed that interaction between nCLU and Bcl-XL resulted in releasing of Bax, promoted apoptosis accompanied by activation of caspase-3 and cytochrome c release (27). Leskov's team (28) discovered that the $\mathrm{N}$ - and C-terminal coiledcoil domain interact with each other, suggesting that this protein could dimerize or fold, however they both produce same contribution. All these results indicated that nCLU is a pro-apoptotic molecule, and the C-terminal coiled-coil domain was the minimal region required for $\mathrm{Ku}$ binding, in additional, Bax is the key molecule in nCLU induced- 
apoptosis mechanism.

Opposite from nCLU, sCLU impedes the activation of Bax by interfering the $\mathrm{Ku}-70-\mathrm{Bax}$ complex in mitochondria, which leads to the release of cytochrome $\mathrm{c}$ and caspase- 3 . $\mathrm{Ku}-70-\mathrm{Bax}$ complex was stabilized as sCLU binds to it in the cytoplasm, suppressing Bax activation and relocation to mitochondria. Moreover, sCLU also cooperated with c-Myc, which confers cancer cells the ability of proliferation and progression in vivo $(14,29)$. Another research revealed that high levels of sCLU upregulated the expression of megalin, also known as low density lipoprotein-related protein 2 (LRP-2) which results in the phosphorylation of Akt. Subsequently, activated Akt caused a decreasing of cytochrome c released by inducing the phosphorylation of Bad. This implicates sCLU PI3K/AKT axis and its receptor megalin protects cancer cells against tumor necrosis factor- $\alpha(\mathrm{TNF}-\alpha)$ induced apoptosis $(30,31)$. Apart from that, $\mathrm{PI} 3 \mathrm{~K} / \mathrm{AKT} / \mathrm{NF}-\kappa \mathrm{B}$ pathway also involved in the matrix metallopeptidase 9 (MMP-9) activation together with ERK1/2 signaling pathway. The authors hypothesis that CLU regulates extracellular matrix (ECM) remodeling through increasing MMP-9 expression in macrophages during tumor cell invasion, inflammation, and/or tissue remodeling (32). Interestingly, through stabilizing the inhibitor $\mathrm{I} \kappa \mathrm{B}$, sCLU regulates $\mathrm{NF}-\kappa \mathrm{B}$ activity in a negative manner, resulting in suppression of tumor cell motility $(33,34)$.

The growth and metastasis of a neoplasm also required formation of adequate vascular support (35). In vitro, Fu's team (36) found out that high levels of sCLU seem to correlate with tumor angiogenesis through inducing the expression of vascular endothelial growth factor (VEGF). Deficiency of sCLU protein after using far-infrared (FIR) radiation or antisense oligonucleotides (ASO's) could lead to effective inhibition of angiogenesis $(37,38)$. Besides that, sCLU was also discovered having activity similar to small heat shock protein, conferring cellular protection against both heat shock and oxidative stress in order to prevent protein precipitation and protect cells from heat and other stresses. To sum up, both heat shock and oxidative stress could induce expression of sCLU mRNA, results in highly sensitive to apoptotic cell death (39-41).

Ubiquitination, sometimes referred as the molecular "kiss of death" for a protein, is a three steps enzymatic process that involves the bonding of a ubiquitin protein to a substrate protein (42). The entire process requires three types of essential enzymes, which is $\mathrm{E} 1$ ubiquitin-activating enzyme, E2 ubiquitin-conjugating enzyme and E3 ubiquitin ligases. This process could affect proteins in many ways, including tagging them for proteasome mediated degradation, promote or prevent protein interaction, alter their location inside a cell (43). There is a study reported that sCLU increases nuclear factor $\kappa \mathrm{B}(\mathrm{NF}-\mathrm{\kappa B})$ nuclear translocation and transcriptional activity by serving as a ubiquitin-binding protein, which could promote survival of prostate cancer cell as a result of enhancing COMMD1 and $\mathrm{I}-\kappa \mathrm{B}$ proteasomal degradation by interacting with members of the SCF-beta-transducin repeat-containing protein (SCF- $\beta$ TrCP) E3 ligase family (44). To sum up, CLU could act as a ubiquitin-like protein to determine the survival of prostate cancer cells. More studies are required to investigate functions of this ubiquitin-like protein and the involved mechanisms in NSCLC.

All these evidences shows that the following are the physiological functions of sCLU: (I) inhibit the activation of Bax by stabilizing Bax-Ku70; (II) activated the PI3K/ AKT survival pathway which promote cancer cells survival;

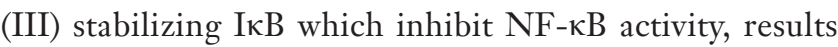
in suppression of tumor cell; (IV) participate in tumor angiogenesis; (V) a cytoprotective chaperone having function similar to small heat shock protein; (VI) plays a role of an ubiquitin-like protein and controls the survival of cancer cells. Molecular function of CLU was illustrated in Figure 1.

\section{$C L U$ and tumorigenesis}

Expression of CLU also been investigated and widely reported overexpression in various types of cancer including gastric cancer (45), prostate (46), breast (47), lung (48) and melanoma (49). High level of sCLU was significantly related to advanced tumor pathological stage and grade in cancer, as well as low recurrence-free survival patients $(50,51)$. Numerous studies also certify that CLU as a promising useful biomarker in different types of cancer including esophageal cancer (52), hepatocellular carcinoma (53), colorectal cancer (54), osteosarcoma (55), ovarian cancer (56), prostate cancer (57) and gastric cancer (58). Using immunohistochemical observation on surgical colon specimens, Pucci et al. (59) discovered the distribution of cytoplasmic CLU was associated with the progression of carcinoma towards high-grade and metastatic, concluded that CLU was related to tumor progression. Another study also reported that CLU could promote the progression of Hepatitis $\mathrm{C}$ virus $(\mathrm{HCV})$-related hepatocellular carcinoma (HCC) by regulating autophagy (60). Therefore, CLU 


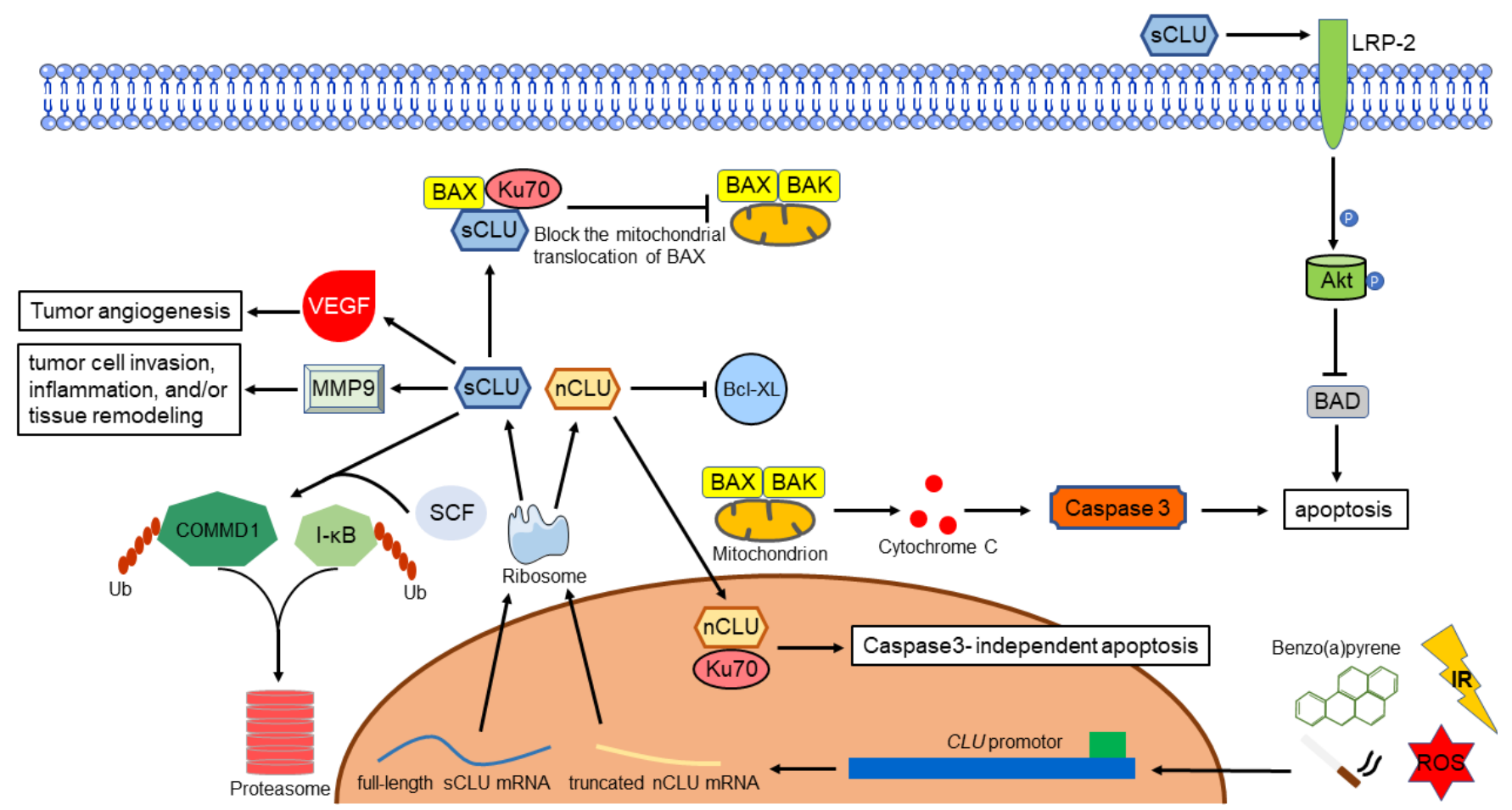

Figure 1 Molecular function of clusterin in tumorgenesis.

overexpression in solid tumor has become a common observation, having the ability to evaluate diagnosis and metastasis potential.

\section{Role of CLU in metastasis}

Apart from relationship with chemoresistance, emerging evidence showed that CLU is overexpressed in several metastatic cancer cells, such as prostate cancer, hepatocellular carcinoma, nasopharyngeal carcinoma and colorectal cancer (61-63) Since CLU plays an important role in cancer metastasis, the mechanisms that CLU favors cancer metastasis have been explored (64). Upregulation of CLU promotes metastasis both in vitro and in vivo by EIF3I/Akt/MMP13 signaling (65). Inhibition of CLU gene using ASO not only could resentisize chemosensitivity of cancer cells, it could also help prevent the growing and metastasis of cancer cells simultaneously (66), also showing that CLU in either serum and/or tissue is expected to be a candidate of diagnosis biomarkers for detection of some early metastatic cancers.

\section{CLU in lung cancer}

\section{Tobacco smoking and CLU}

Tobacco smoking remains the predominant risk factor for the development of lung cancer. According to the Global Health Observatory (GHO) data, there were over 1.1 billion people who smoked tobacco in 2015 (67). In China, World Health Organization (WHO) estimates about $27 \%$ of China's population smoked (approximately $303,926,600$ persons) in year 2010 and around $24 \%$ of the population (approximately $291,267,700$ persons) will be smokers by 2025 . Although the population of smokers seems to be decreasing in 15 years, still, the mean number of cigarettes consume daily per smoker and the absolute number of total deaths due to smoking increased over time. In additional, trend of initiation to smoke started at a very young age, which is also becoming a concern issue (68-70). Recently, a study discovered a connection between CLU and tobacco smoking, although the concentration of CLU did not correlate with nicotine addiction or dependence scores, but the significantly increased of CLU was found 
in the saliva of prolonged tobacco and high intensity of tobacco consumption users, moreover the levels of CLU protein decreased significantly in 6 months after smoking cessation (71). Another study also found out that tobacco cessation could improve the overall survival of lung cancer patients (72).

Benzo(a)pyrene $(\mathrm{BaP})$ is a ubiquitous environment contaminant found in coal tar, automobile exhausts fumes, tobacco smoke and charcoal grilled food, it has been reported as one of the components in cigarette mainstream smoke (73). Overexpression of CLU, neuropilin-2 (NRP2) and A-kinase anchor protein 12 (AKAP12) have been identified in $\mathrm{BaP}$-transformed $16 \mathrm{HBE}$ cell line T-16HBE-C1 cells (74). Similar results also obtained from another research. Levels of CLU and NRP2 were significant evaluated in culture supernatant of T-16HBE-C1 xenografted nude mice compared with control. Although CLU and NRP2 could predicate the progression of tumor respectively, however, CLU appeared to be more sensitive than NRP2 (75).

These results implying that $\mathrm{BaP}$ could be one of the factors inducing expression of salivary CLU during smoking, additionally tobacco cessation may be helpful in the prognosis of lung cancer. Despite that, the mechanisms between $\mathrm{BaP}$ and other composition in the tobacco and CLU still needed to be probed.

\section{CLU as a tumor biomarker}

NSCLC accounts for $85 \%$ of primary lung cancers, among three of the histological subtypes mentioned before, adenocarcinoma is the most common one (76). By using the combination of proteomic study and bioinformatic prediction on signal peptides, CLU also served as a solid serological biomarker in lung adenocarcinoma, together with Calsyntenin-1 (CLSTN1) and neutrophil gelatinaseassociated lipocalin (NGAL) (77). For early stages NSCLC patients, surgery is still remaining the cornerstone of treatment and recommended by surgeons $(4,78)$. Expression of CLU was proven to be a useful biomarker and related to fewer relapses and longer survival in surgically resected lung adenocarcinoma. Panico et al. (20) also discovered the decreasing expression of CLU from well-differentiated to poorly differentiated adenocarcinomas.

\section{Distribution of CLU in NSCLC tissue}

In order to determine the location and distribution of
CLU in lung cancer, Jeffrey $M$ and colleagues stained the specimens retrieved from lung cancer patients with anticlusterin $\alpha$-chain antibody, an antibody used to detect both nuclear and cytoplasmic isoform. Together, they observed cytoplasmic CLU staining from all the 44 samples, and none of the nuclear staining was observed. Furthermore, cCLU staining was associated with longer survival in patients with surgically resected NSCLC which is similar to the study mentioned previously (79). Interestingly, another research concluded that both nuclear and cCLU staining was observed in lung cancer, CLU staining only observed in 70 patients $(57.9 \%)$, nuclear staining only in 27 patients $(22.3 \%)$ and both nuclear and cytoplasmic staining in 16 patients (13.2\%). In additional, lung adenocarcinomas were more likely to have cytoplasm staining only (80). The different results obtained from both studies may be due to lacking of larger sample size, sampling deviation and value deviation.

\section{CLU and lung cancer chemotherapy}

Platinum-based chemotherapy remains the standard treatments for advanced NSCLC patients. The platinum compounds currently used are cisplatin and carboplatin. However, limited efficacy of chemotherapy is still one of the major impediments in the treatment of NSCLC. Studies have found out that high levels of sCLU expressed in various cancer, including breast and ovary, is associated with chemoresistance (81-85). In lung cancer, researches carried out in animal models and lung cancer cell lines, revealing that expression of sCLU is upregulated after exposure to chemotherapy and radiotherapy (86). The overexpression of sCLU confers resistance to cisplatin (DDP) in A549 cells, and by silencing it could resensitize A549 cells to DDP through AKT and ERK1/2 pathway in vitro (87). Using luciferase tests, another research also shows that miR-195 could bound to the 3'-UTR of CLU. With overexpression of miR-195, amassment of CLU could be reduced, further improved the sensitivity of cancer cells which is resistant to docetaxel (88). Chen's study (89) also discovered that sCLU is related to the development of chemoresistance to DDP. By using Targetscan and luciferase assay, they found out miR-378 could directly targeted to sCLU, decrease sCLU expression in lung adenocarcinoma cells, thus enhancing cell apoptosis and resensitize to cDDP both in vitro and in vivo. Since numerous studies revealed that inhibition of CLU could increase the effectiveness of chemotherapeutic agents to kill tumor cells $(48,90)$, the selective silencing of 
$C L U$ gene may be reasonable.

\section{Treatment of NSCLC with ASO against CLU}

Advances in the field of nucleic acid chemistry hold potential for developing gene silencers, which may help mediating tumor progression and treatment resistance. ASO-based agents are synthetic fragments of DNA, specifically hybridize with complementary mRNA regions of a target gene organized by Ago2 to form RNA/DNA duplexes, therefore inhibit gene expression (91). The combination of an ASO with other compounds, such as therapeutic agents, has shown synergistic antineoplastic effects (92-94). In particular, custirsen (OGX-011), a second-generation ASO, inhibitor of $C L U$ gene could be an attractive approach for treatment $(95,96)$. In lung cancer models, CLU gene suppressed by custirsen also proven enhanced sensitivity to chemotherapies such as paclitaxel and gemcitabine both in vitro and in vivo. The enhanced chemosensitivity of A549 cell line towards paclitaxel increased in a dose-dependent manner after ASO treatment, significantly reducing cell viability. CLU ASO also enhanced micellar paclitaxel and gemcitabine chemosensitivity in A549 xenograft in both nude and SCID mice, causing a $54 \%$ and $60 \%$ reduction in mean tumor volume by 5 weeks following initiation of treatment (48).

With support of preclinical trail results, custirsen was administered in combination with a gemcitabine and platinum regimen in phase I/II trail of advanced nonsmall cell lung cancer, showing improvement in survival data. Notably, the estimated ratio of death hazard for patients having a CLU response to the death hazard rate for those not having a response was 0.5 , representing a $50 \%$ reduction in the hazard of death with CLU response (97). Similar results also obtained previously in advanced prostatic cancer resistant patients, in which phase III has been completed in 2015 (98,99). Clinical studies conducted related to CLU and OGX-011 are summarized in Table 1.

\section{CLU and NSCLC radiotherapy}

Apart from chemotherapy, role of CLU on radiation sensitivity also has been investigating for years. Interestingly, similar results were obtained, CLU is frequently overexpressed, a protein which could significantly decrease radiotherapy sensitivity in many human cancers $(46,86,100)$.

The generation of reactive oxygen species (ROS) is the postulated mechanism of action for radiation therapy. During water radiolysis, DNA lesions caused by ROS react with oxygen to form stable DNA peroxides, enhancing the efficacy of radiotherapy (101). Sensitivity of cancer cells towards radiotherapy decreased may due to the cytoprotective role function of CLU, which was stimulated when exposing to oxidative stress as stated before. High levels of CLU could act as a cell survival protein that mediates radioresistance through the inhibition of apoptosis (102). Sensitivity of cancer cells towards radiotherapy resensitized after the inhibition of CLU gene by using ASOs (103). Another research demonstrated that combination treatment of radiotherapy and OGX-011 could greatly decrease survival of lung cancer cells, showing that CLU may be a therapeutic target in radiotherapy (104). Moreover, Watari et al. (105) demonstrated that CLU could also be used as a molecular marker to predict overall survival of advanced-stage cancer patients with curative intended radiotherapy.

\section{CLU and NSCLC epithelial-mesenchymal transition}

Epithelial-mesenchymal transition (EMT) is a process by which epithelial cells lose their cell polarity and cellcell adhesion, therefore gaining the ability to migrate and invade as single cells (106). The ectopic expression of forkhead box P3 (FOXP3), also known as scurfin, was found inducing EMT and activated Wnt/ $\beta$-catenin signaling pathway, results in promoting tumor growth and metastasis of NSCLC (107).

Numerous studies regarding the relationship between CLU and EMT have been carried out. Transcriptome profiling of a TGF- $\beta$-induced EMT demonstrated by Lenferink et al. (108) revealed that upregulated levels of sCLU plays an important role in promoting EMT. Shiota's team (109) discovered that TGF- $\beta$-induced CLU expression was mediated by Twist 1 through binding to distal promoter of CLU, they demonstrated that treatment with EMTinducing cytokine TGF- $\beta$ could unregulated the expression of Twist 1 followed by CLU expression. Another study discovered that CLU interact with eHSP90 $\alpha$, together they synergistically promote the EMT process. By going through proximity ligation assay and co-immunoprecipitation experiments, the authors showed that CLU take part in eHSP90 $\alpha$-LRP1 complex formation by increasing the binding affinity of eHSP90 $\alpha$ and its receptor, low-density lipoprotein receptor-related protein 1 (LRP1) (110). These conclude that both TGF- $\beta$-induced CLU expression, interaction of CLU and eHSP90 $\alpha$-LRP1 complex could 

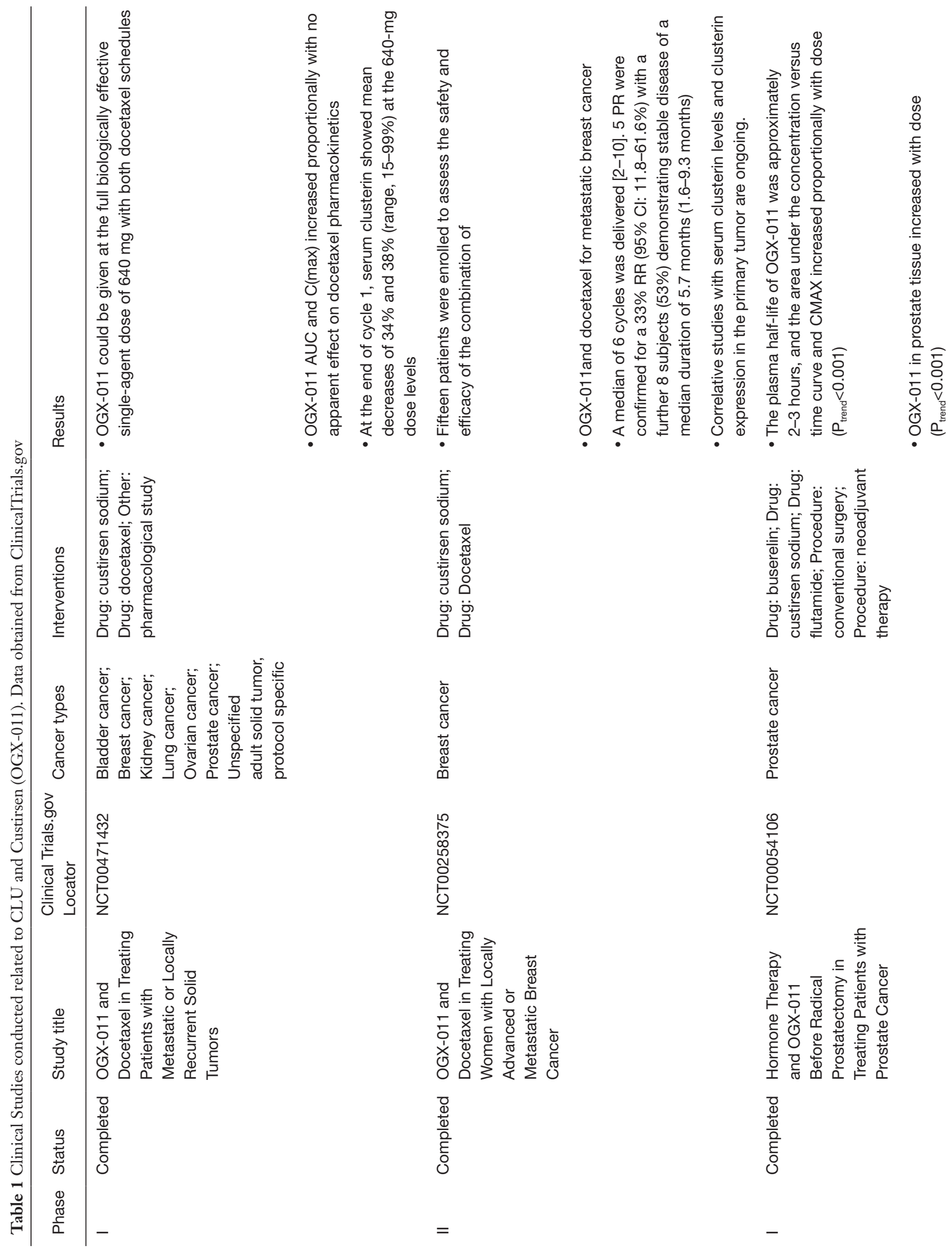

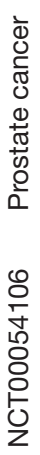
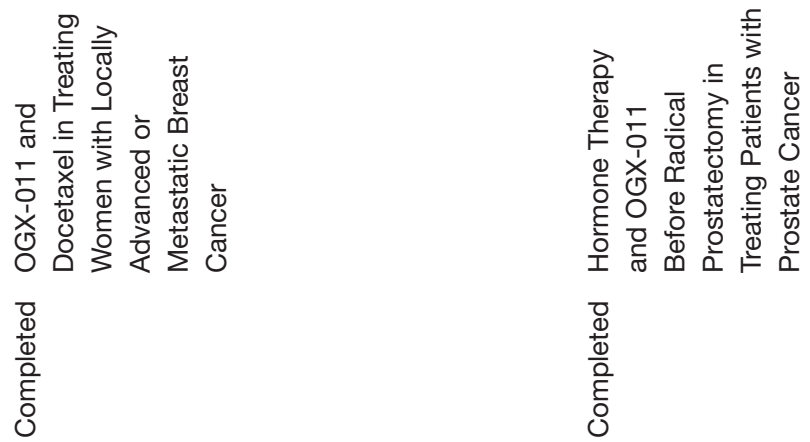


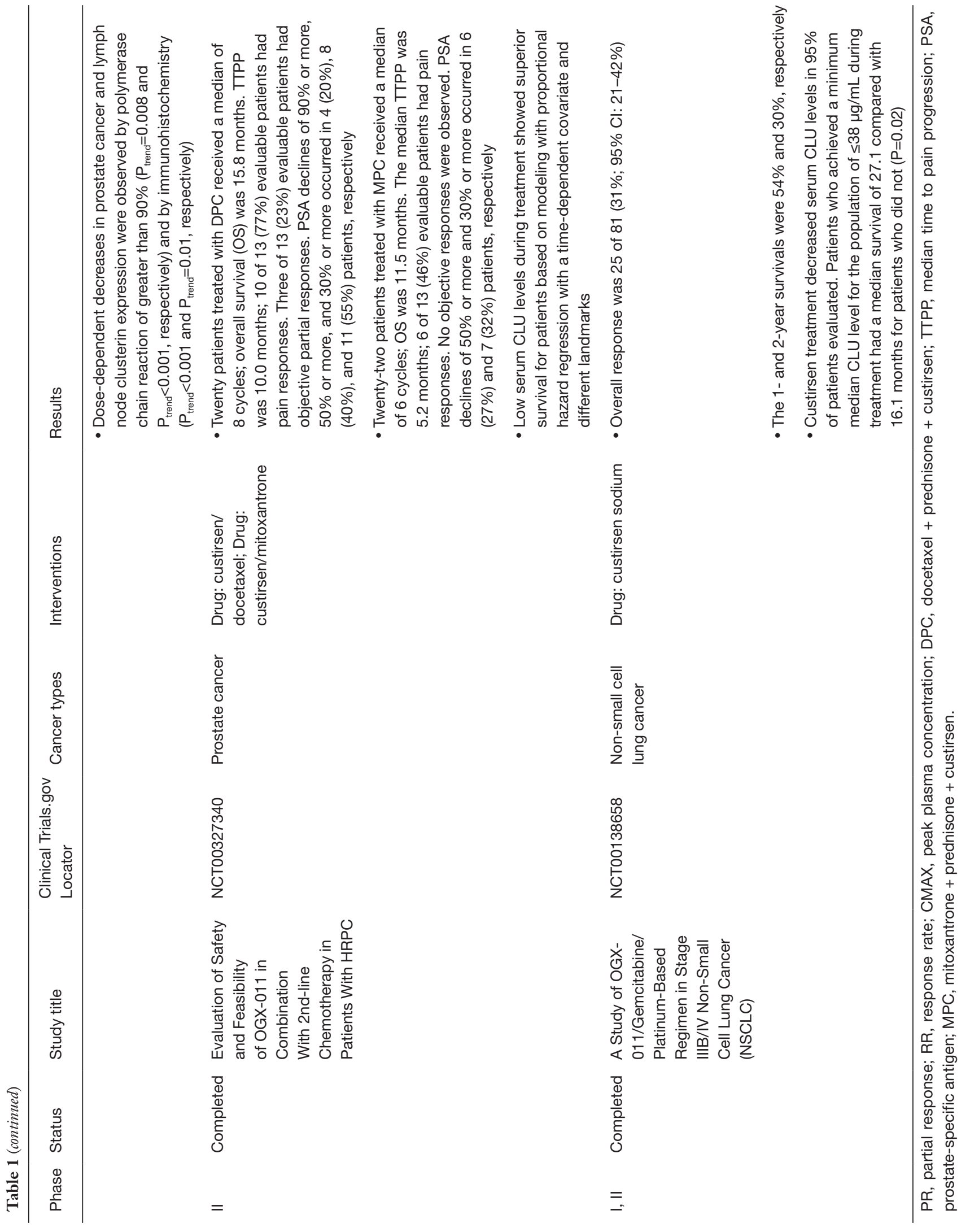


promote EMT, but there is no relevant research between these two mechanisms to determine their association.

In lung cancer, CLU was observed modulating transdifferentiation of lung squamous cell carcinoma to lung adenocarcinoma by promoting epithelial-mesenchymal transition (EMT) (111). The invasiveness of lung adenocarcinoma could also be attributed to CLU-mediates EMT through modulating ERK signalling and Slug expression (18). Moreover, siRNA-mediated knockdown of PTEN, a target of miR-19, also resulted in EMT, migration, and invasion of lung cancer cells, suggesting that PTEN is also involved (112).

SRAMs refers to genes that significantly repressed in association with DNA methylation, Lin's team (113) integrated the gene expression profiles involved in migration and metastasis of NSCLC, they found out that the EMT-SRAMs was related and also associated with erlotinib resistance in epithelial growth factor receptor (EGFR) NSCLC cell lines. Cell-based studies carried out, demonstrated that increasing expression of the E-cadherin in cancer cells, the epithelial cell marker, are more sensitive to EGFR inhibitor, erlotinib (114). Correlation between cancer cells sensitivity to erlotinib and E-cadherin expression was discovered, it has been shown that restoration of E-cadherin expression increases sensitivity to erlotinib (115). E-cadherin is a cell adhesion molecule that plays a key role in the signaling and regulation of EMT. Decreasing expression of E-cadherin is associated with increasing of EMT $(116,117)$. When NSCLC cell lines expressing sCLU was treated with anti-CLU antibody, the expression of E-cadherin increased. In parallel, inhibition of EMT and decreasing of matrix metalloproteinase-2 (MMP2) gene, could inhibit the invasion of cancer (118-120). Moreover, high cell surface E-cadherin are more sensitive to radiation. Theys's team (121) found out that EMT-like conversion of mesenchymal phenotype could promote radioresistance in human tumor cells. Thus E-cadherin expression varies in tumors induced by changes in microenvironment such as CLU or other stimuli may contribute to the sensitivity of tumor cells to radiotherapy and chemotherapy. Furthermore, BaP, one of the cigarette's mainstream smoke components as mentioned before, also found taking part in the induction of EMT (122). By using quantitative real-time PCR, BaP was observed could elevated the expression levels of linc00673 in an aryl hydrocarbon receptor- (AHR) dependent manner, as a result of E-cadherin and MMP-2 expression inhibition. Therefore, promote lung cancer cells migration, invasion and EMT (123). However, there is no evidence proving that $\mathrm{BaP}$ and CLU work synergistically.

\section{CLU, EMT and PD-L1 in NSCLC}

Recently, immunotherapy regarding programmed cell death 1 programmed death ligand 1 (PD-1/PD-L1) has emerged as a hot topic. PD-L1 positive rate was much higher in patients with mesenchymal phenotypes, especially EGFRmutated pulmonary adenocarcinomas (pADC) compared to epithelial phenotypes, indicating that mesenchymal phenotypes patients are more likely to benefits from PD-1/ PD-L1 blocking immunotherapy (124). The expression of PD-L1 was proven dependent of EMT, regulated by TGF $-\alpha$ and TGF- $\beta$ synergistically through NF- $\kappa \mathrm{B}$ and DNA methylation (125). Co-expression of PD-L1 with EMT transition of circulating tumor cells (CTCs) was associated with poor survival in patients which undergo curatively resected NSCLC (126). In addition, Raimondi et al. (127) hypothesized that PD-L1 expression and EMT markers might represent NSCLC cells a possible molecular background for immune escape. These studies together indicate that CLU and EMT together participated in the metastasis of lung cancer, it is also possible that PD-L1 was also involved. Mechanism of tumor-intrinsic regulation of PD-L1 has been discovered in lung cancer which linked EMT to cytotoxic T cells dysfunction and metastasis (128). However, the relationship between CLU and PD-L1 remained unknown, therefore further investigation into the relationship between CLU and PD-L1 is needed.

\section{Conclusions}

CLU is a protein widely exists in almost all physiological fluids. Various stresses such as tobacco smoking, oxidative stress, inflammation, stress response could increase the expression of CLU. Overexpression of CLU has been confirmed in many malignancies, including NSCLC. In this review, we have shown that CLU participate in many phases of NSCLC tumorigenesis, including cancer cell survival, apoptosis, tumor angiogenesis and metastasis.

The ubiquitin-like function of CLU proven could improve prostate cancer cell survival, yet, the influence on lung cancer cells ought to be probed.

In NSCLC, high level of CLU subsequently triggers downstream pathway resulting in insensitivity to chemotherapy or radiotherapy. Thus, CLU has also been proposed as a potential prognostic biomarker and 
therapeutic target. Several RCTs have proved the efficiency of anti-CLU treatment in NSCLC. By treating with the second-generation ASO Custirsen, chemosensitivity and radiosensitivity of lung cancer patients could be enhanced.

All these together, shows that CLU is an important protein which is related with tumorigenesis and treatment of NSCLC. In the subsequent studies, its biological function such as ubiquitin-like function, as well as the association between CLU and immunotherapy and targeted therapy is worth exploring.

\section{Acknowledgments}

Funding: This study was supported by the grant from The National Natural Science Foundation (81871882, 82072557); Outstanding Academic Leader of Shanghai (20XD1402300); Shanghai Sailing Program (21YF1427100) and Shanghai Municipal Education Commission-Gaofeng Clinical Medicine Grant Support (20172005).

\section{Footnote}

Reporting Checklist: The authors have completed the Narrative Review reporting checklist. Available at http:// dx.doi.org/10.21037/tlcr-20-1298

Peer Review File: Available at http://dx.doi.org/10.21037/ tlcr-20-1298

Conflicts of Interest: All authors have completed the ICMJE uniform disclosure form (available at http://dx.doi. org/10.21037/tlcr-20-1298). The authors have no conflicts of interest to declare.

Ethical Statement: The authors are accountable for all aspects of the work in ensuring that questions related to the accuracy or integrity of any part of the work are appropriately investigated and resolved.

Open Access Statement: This is an Open Access article distributed in accordance with the Creative Commons Attribution-NonCommercial-NoDerivs 4.0 International License (CC BY-NC-ND 4.0), which permits the noncommercial replication and distribution of the article with the strict proviso that no changes or edits are made and the original work is properly cited (including links to both the formal publication through the relevant DOI and the license). See: https://creativecommons.org/licenses/by-nc-nd/4.0/.

\section{References}

1. Ferlay J, Colombet M, Soerjomataram I, et al. Estimating the global cancer incidence and mortality in 2018: GLOBOCAN sources and methods. Int J Cancer 2019;144:1941-53.

2. American Cancer Society key-statistics for lung cancer. Available online: https://www.cancer.org/cancer/lungcancer/about/key-statistics.html

3. Travis WD, Brambilla E, Nicholson AG, et al. The 2015 World Health Organization classification of lung tumors: impact of genetic, clinical and radiologic advances since the 2004 classification. J Thorac Oncol 2015;10:1243-60.

4. Postmus PE, Kerr KM, Oudkerk M, et al. Early and locally advanced non-small-cell lung cancer (NSCLC): ESMO Clinical Practice Guidelines for diagnosis, treatment and follow-up. Ann Oncol 2017;28:iv1-21.

5. Torre LA, Siegel RL, Jemal A. Lung cancer statistics. In: Lung cancer and personalized medicine. Springer, Cham; 2016.

6. Byers LA, Rudin CM. Small cell lung cancer: where do we go from here? Cancer 2015;121:664-72.

7. Palma DA, Warner A, Louie AV, et al. Thoracic radiotherapy for extensive stage small-cell lung cancer: a meta-analysis. Clin Lung Cancer 2016;17:239-44.

8. Barnes H, See K, Barnett S, et al. Surgery for limitedstage small-cell lung cancer. Cochrane Database Syst Rev 2017;4:CD011917.

9. Montpetit ML, Lawless KR, Tenniswood M. Androgenrepressed messages in the rat ventral prostate. Prostate 1986;8:25-36.

10. Jenne DE, Tschopp J. Clusterin: the intriguing guises of a widely expressed glycoprotein. Trends Biochem Sci 1992;17:154-9.

11. Jenne DE, Tschopp J. Molecular structure and functional characterization of a human complement cytolysis inhibitor found in blood and seminal plasma: identity to sulfated glycoprotein 2, a constituent of rat testis fluid. Proc Natl Acad Sci U S A 1989;86:7123-7.

12. Shannan B, Seifert M, Boothman DA, et al. Clusterin and DNA repair: a new function in cancer for a key player in apoptosis and cell cycle control. J Mol Histol 2006;37:183-8.

13. Nguan CYC, Guan Q, Gleave ME, et al. Promotion of cell proliferation by clusterin in the renal tissue repair phase after ischemia-reperfusion injury. Am J Physiol Renal Physiol 2014;306:F724-33.

14. Zhang H, Kim JK, Edwards CA, et al. Clusterin inhibits 
apoptosis by interacting with activated Bax. Nat Cell Biol 2005;7:909-15.

15. Cunin P, Beauvillain C, Miot C, et al. Clusterin facilitates apoptotic cell clearance and prevents apoptotic cellinduced autoimmune responses. Cell Death Dis 2016;7:e2215.

16. Fu Y, Lai Y, Liu J, et al. Lentivirus-mediated shRNA interference of clusterin blocks proliferation, motility, invasion and cell cycle in the ovarian cancer cells. J Ovarian Res 2015;8:59.

17. Cheng CY, Cherng SH, Wu WJ, et al. Regulation of chemosensitivity and migration by clusterin in non-small cell lung cancer cells. Cancer Chemother Pharmacol 2012;69:145-54.

18. Chou TY, Chen WC, Lee AC, et al. Clusterin silencing in human lung adenocarcinoma cells induces a mesenchymalto-epithelial transition through modulating the ERK/Slug pathway. Cell Signal 2009;21:704-11.

19. Liu W, Cai L, Ning J, et al. Expression and significance of clusterin in Anip973/NVB cell lines. Zhongguo Fei Ai Za Zhi 2008;11:696-9.

20. Panico F, Casali C, Rossi G, et al. Prognostic role of clusterin in resected adenocarcinomas of the lung. Lung Cancer 2013;79:294-9.

21. Tellez T, Garcia-Aranda M, Redondo M. The role of clusterin in carcinogenesis and its potential utility as therapeutic target. Curr Med Chem 2016;23:4297-308.

22. de Silva HV, Harmony JA, Stuart WD, et al. Apolipoprotein J: structure and tissue distribution. Biochemistry 1990;29:5380-9.

23. Stewart EM, Aquilina JA, Easterbrook-Smith SB, et al. Effects of glycosylation on the structure and function of the extracellular chaperone clusterin. Biochemistry 2007;46:1412-22.

24. Jones SE, Jomary C. Clusterin. Int J Biochem Cell Biol 2002;34:427-31.

25. Bettuzzi S, Troiano L, Davalli P, et al. In vivo accumulation of sulfated glycoprotein 2 mRNA in rat thymocytes upon dexamethasone-induced cell death. Biochem Biophys Res Commun 1991;175:810-5.

26. Yang CR, Leskov K, Hosley-Eberlein K, et al. Nuclear clusterin/XIP8, an $\mathrm{x}$-ray-induced $\mathrm{Ku}$ 70-binding protein that signals cell death. Proc Natl Acad Sci U S A 2000;97:5907-12.

27. Kim N, Yoo J C, Han J Y, et al. Human nuclear clusterin mediates apoptosis by interacting with Bcl-XL through C-terminal coiled coil domain. J Cell Physiol 2012;227:1157-67.
28. Leskov KS, Klokov DY, Li J, et al. Synthesis and functional analyses of nuclear clusterin, a cell death protein. J Biol Chem 2003;278:11590-600.

29. Trougakos IP, Lourda M, Antonelou MH, et al. Intracellular clusterin inhibits mitochondrial apoptosis by suppressing $\mathrm{p} 53$-activating stress signals and stabilizing the cytosolic Ku70-Bax protein complex. Clin Cancer Res 2009;15:48-59.

30. Ammar H, Closset JL. Clusterin activates survival through the phosphatidylinositol 3-kinase/Akt pathway. J Biol Chem 2008;283:12851-61.

31. Wang Y, Wang X, Zhao H, et al. Clusterin confers resistance to TNF-alpha-induced apoptosis in breast cancer cells through NF-kappaB activation and Bcl-2 overexpression. J Chemother 2012;24:348-57.

32. Shim YJ, Kang BH, Jeon HS, et al. Clusterin induces matrix metalloproteinase-9 expression via ERK1/2 and PI3K/Akt/NF-kB pathways in monocytes/macrophages. J Leukoc Biol 2011;90:761-9.

33. Santilli G, Aronow BJ, Sala A. Essential requirement of apolipoprotein $\mathrm{J}$ (clusterin) signaling for ІкB expression and regulation of NF- $\mathrm{KB}$ activity. J Biol Chem 2003;278:38214-9.

34. Rizzi F, Bettuzzi S. The clusterin paradigm in prostate and breast carcinogenesis. Endocr Relat Cancer 2010;17:R1.

35. Herbst RS, Fidler IJ. Angiogenesis and lung cancer: potential for therapy. Clin Cancer Res 2000;6:4604-6.

36. Fu Y, Lai Y, Wang Q, et al. Overexpression of clusterin promotes angiogenesis via the vascular endothelial growth factor in primary ovarian cancer. Mol Med Rep 2013;7:1726-32.

37. Hwang S, Lee DH, Lee IK, et al. Far-infrared radiation inhibits proliferation, migration, and angiogenesis of human umbilical vein endothelial cells by suppressing secretory clusterin levels. Cancer Lett 2014;346:74-83.

38. Jackson JK, Gleave ME, Gleave J, et al. The inhibition of angiogenesis by antisense oligonucleotides to clusterin. Angiogenesis 2005;8:229-38.

39. Viard I, Wehrli P, Jornot L, et al. Clusterin gene expression mediates resistance to apoptotic cell death induced by heat shock and oxidative stress. J Invest Dermatol 1999;112:290-6.

40. Humphreys DT, Carver JA, Easterbrook-Smith SB, et al. Clusterin has chaperone-like activity similar to that of small heat shock proteins. J Biol Chem 1999;274:6875-81.

41. Carver JA, Rekas A, Thorn DC, et al. Small heat-shock proteins and clusterin: intra-and extracellular molecular chaperones with a common mechanism of action and 
function? IUBMB life 2003;5 5:661-8.

42. News Medical Life Sciences. Available online: https:// www.news-medical.net/life-sciences/Ubiquitination(Ubiquitylation).aspx

43. Hochstrasser M. Origin and function of ubiquitin-like proteins. Nature 2009;458:422-9.

44. Zoubeidi A, Ettinger S, Beraldi E, et al. Clusterin facilitates COMMD1 and I- $\kappa \mathrm{B}$ degradation to enhance NF- $\kappa \mathrm{B}$ activity in prostate cancer cells. Mol Cancer Res 2010;8:119-30.

45. Mu L, Yang F, Guo D, et al. Overexpression of secretory clusterin (sCLU) induces chemotherapy resistance in human gastric cancer cells by targeting miR-195-5p. Bioengineered 2020;11:472-83.

46. Zellweger T, Kiyama S, Chi K, et al. Overexpression of the cytoprotective protein clusterin decreases radiosensitivity in the human $\mathrm{LNCaP}$ prostate tumour model. BJU Int 2003;92:463-9.

47. Yom CK, Woo HY, Min SY, et al. Clusterin overexpression and relapse-free survival in breast cancer. Anticancer Res 2009;29:3909-12.

48. July LV, Beraldi E, So A, et al. Nucleotide-based therapies targeting clusterin chemosensitize human lung adenocarcinoma cells both in vitro and in vivo. Mol Cancer Ther 2004;3:223-32.

49. Mustafi S, Sant DW, Liu ZJ, et al. Ascorbate induces apoptosis in melanoma cells by suppressing Clusterin expression. Sci Rep 2017;7:3671.

50. Kurahashi T, Muramaki M, Yamanaka K, et al. Expression of the secreted form of clusterin protein in renal cell carcinoma as a predictor of disease extension. BJU Int 2005;96:895-9.

51. Miyake H, Yamanaka K, Muramaki M, et al. Enhanced expression of the secreted form of clusterin following neoadjuvant hormonal therapy as a prognostic predictor in patients undergoing radical prostatectomy for prostate cancer. Oncol Rep 2005;14:1371-5.

52. Guo W, Ma X, Xue C, et al. Serum clusterin as a tumor marker and prognostic factor for patients with esophageal cancer. Dis Markers 2014;2014:168960.

53. Nafee AM, Pasha HF, Abd El Aal SM, et al. Clinical significance of serum clusterin as a biomarker for evaluating diagnosis and metastasis potential of viralrelated hepatocellular carcinoma. Clin Biochem 2012;45:1070-4.

54. Artemaki PI, Sklirou A D, Kontos CK, et al. High clusterin (CLU) mRNA expression levels in tumors of colorectal cancer patients predict a poor prognostic outcome. Clin
Biochem 2020;75:62-9.

55. Ma J, Gao W, Gao J. sCLU as prognostic biomarker and therapeutic target in osteosarcoma. Bioengineered 2019;10:229-39.

56. Lyu N, Wang Y, Wang J, et al. Study on early diagnosis of epithelial ovarian cancer by analysis of plasma septin- 9 and clusterin level. J Cancer Res Ther 2018;14:S444.

57. Blumenstein B, Saad F, Hotte S, et al. Reduction in serum clusterin is a potential therapeutic biomarker in patients with castration-resistant prostate cancer treated with custirsen. Cancer Med 2013;2:468-77.

58. Bi J, Guo AL, Lai YR, et al. Overexpression of clusterin correlates with tumor progression, metastasis in gastric cancer: a study on tissue microarrays. Neoplasma 2010;57:191.

59. Pucci S, Bonanno E, Pichiorri F, et al. Modulation of different clusterin isoforms in human colon tumorigenesis. Oncogene 2004;23:2298-304.

60. Fu N, Du H, Li D, et al. Clusterin contributes to hepatitis $\mathrm{C}$ virus-related hepatocellular carcinoma by regulating autophagy. Life Sci 2020;256:117911.

61. Shiota M, Zardan A, Takeuchi A, et al. Clusterin mediates TGF- $\beta$-induced epithelial-mesenchymal transition and metastasis via Twist1 in prostate cancer cells. Cancer Res 2012;72:5261-72.

62. Li Y, Lu J, Zhou S, et al. Clusterin induced by N, N'Dinitrosopiperazine is involved in nasopharyngeal carcinoma metastasis. Oncotarget 2016;7:5548.

63. Zhu Y, Chen P, Gao Y, et al. MEG3 activated by vitamin D inhibits colorectal cancer cells proliferation and migration via regulating clusterin. EBioMedicine 2018;30:148-57.

64. Wang C, Jiang K, Kang X, et al. Tumor-derived secretory clusterin induces epithelial-mesenchymal transition and facilitates hepatocellular carcinoma metastasis. Int J Biochem Cell Biol 2012;44:2308-20.

65. Wang C, Jin G, Jin H, et al. Clusterin facilitates metastasis by EIF3I/Akt/MMP13 signaling in hepatocellular carcinoma. Oncotarget 2015;6:2903.

66. Miyake H, Hara I, Kamidono S, et al. Synergistic chemsensitization and inhibition of tumor growth and metastasis by the antisense oligodeoxynucleotide targeting clusterin gene in a human bladder cancer model. Clin Cancer Res 2001;7:4245-52.

67. World Health Organization (WHO) Global Health Observatory (GHO) data. Available online: https://www. who.int/gho/tobacco/use/en/

68. World Health Organization. WHO global report on trends in prevalence of tobacco smoking 2015. World 
Health Organization, 2015.

69. Li S, Meng L, Chiolero A, et al. Trends in smoking prevalence and attributable mortality in China, 1991-2011. Prev Med 2016;93:82-7.

70. Parascandola M, Xiao L. Tobacco and the lung cancer epidemic in China. Transl Lung Cancer Res 2019;8:S21.

71. Pallardo-Fernández I, Iglesias V, Rodríguez-Rivera C, et al. Salivary clusterin as a biomarker of tobacco consumption in nicotine addicts undergoing smoking cessation therapy. J Smok Cessat 2020;15:171-4.

72. Dobson Amato KA, Hyland A, Reed R, et al. Tobacco cessation may improve lung cancer patient survival. J Thorac Oncol 2015;10:1014-9.

73. Smith CJ, Perfetti TA, Rumple MA, et al. "IARC group 2A Carcinogens" reported in cigarette mainstream smoke. Food and chemical toxicology 2000;38:371-83.

74. Zhao P, Fu J, Yao B, et al. Label-free quantitative proteomic analysis of benzo(a)pyrene-transformed $16 \mathrm{HBE}$ cells serum-free culture supernatant and xenografted nude mice sera. Chem Biol Interact 2016;245:39-49.

75. Wang Y, Jia Y, Yan L, et al. Clusterin and neuropilin-2 as potential biomarkers of tumor progression in benzo [a] pyrene-transformed $16 \mathrm{HBE}$ cells xenografted nude mouse model. Chem Biol Interact 2017;275:145-51.

76. Cheng TYD, Cramb S M, Baade P D, et al. The international epidemiology of lung cancer: latest trends, disparities, and tumor characteristics. J Thorac Oncol 2016;11:1653-71.

77. Chu Y, Lai YH, Lee MC, et al. Calsyntenin-1, clusterin and neutrophil gelatinase-associated lipocalin are candidate serological biomarkers for lung adenocarcinoma. Oncotarget 2017;8:107964.

78. Ettinger DS, Wood DE, Aggarwal C, et al. NCCN Guidelines Insights: Non-Small Cell Lung Cancer, Version 1.2020. J Natl Compr Canc Netw 2019;17:1464-72.

79. Albert JM, Gonzalez A, Massion PP, et al. Cytoplasmic clusterin expression is associated with longer survival in patients with resected non-small cell lung cancer. Cancer Epidemiol Biomarkers Prev 2007;16:1845-51.

80. Li H, Liu S, Zhu XL, et al. Clusterin immunoexpression and its clinical significance in patients with non-small cell lung cancer. Lung 2010;188:423-31.

81. Koltai T. Clusterin: a key player in cancer chemoresistance and its inhibition. Onco Targets Ther 2014;7:447.

82. Djeu JY, Wei S. Clusterin and chemoresistance. Adv Cancer Res 2009;105:77-92.

83. Hassan MK, Watari H, Christenson L, et al. Intracellular clusterin negatively regulates ovarian chemoresistance: compromised expression sensitizes ovarian cancer cells to paclitaxel. Tumour Biol 2011;32:1031.

84. Park DC, Yeo SG, Wilson MR, et al. Clusterin interacts with Paclitaxel and confer Paclitaxel resistance in ovarian cancer. Neoplasia 2008;10:964-72.

85. So A, Sinnemann S, Huntsman D, et al. Knockdown of the cytoprotective chaperone, clusterin, chemosensitizes human breast cancer cells both in vitro and in vivo. Mol Cancer Ther 2005;4:1837-49.

86. Panico F, Rizzi F, Fabbri LM, et al. Clusterin (CLU) and lung cancer. Adv Cancer Res 2009;105:63-76.

87. Zhang B, Zhang K, Liu Z, et al. Secreted clusterin gene silencing enhances chemosensitivity of a549 cells to cisplatin through AKT and ERK1/2 pathways in vitro. Cell Physiol Biochem 2014;33:1162-75.

88. Ma X, Zou L, Li X, et al. MicroRNA-195 regulates docetaxel resistance by targeting clusterin in prostate cancer. Biomed Pharmacother 2018;99:445-50.

89. Chen $X$, Jiang $Y$, Huang Z, et al. miRNA-378 reverses chemoresistance to cisplatin in lung adenocarcinoma cells by targeting secreted clusterin. Sci Rep 2016;6:19455.

90. Lee CH, Jin RJ, Kwak C, et al. Suppression of clusterin expression enhanced cisplatin-induced cytotoxicity on renal cell carcinoma cells. Urology 2002;60:516-20.

91. Crooke ST. Molecular mechanisms of antisense oligonucleotides. Nucleic Acid Ther 2017;27:70-7.

92. Zellweger T, Miyake H, July LV, et al. Chemosensitization of human renal cell cancer using antisense oligonucleotides targeting the antiapoptotic gene clusterin. Neoplasia 2001;3:360.

93. Leung S, Miyake H, Zellweger T, et al. Synergistic chemosensitization and inhibition of progression to androgen independence by antisense Bcl-2 oligodeoxynucleotide and paclitaxel in the $\mathrm{LNCaP}$ prostate tumor model. Int J Cancer 2001;91:846-50.

94. Miyake H, Eto H, Hara I, et al. Synergistic antitumor activity by combined treatment with gemcitabine and antisense oligodeoxynucleotide targeting clusterin gene in an intravesical administration model against human bladder cancer kotcc-1 cells. J Urol 2004;171:2477-81.

95. Muramaki M, So A, Hayashi N, et al. Chemosensitization of gemcitabine-resistant human bladder cancer cell line both in vitro and in vivo using antisense oligonucleotide targeting the anti-apoptotic gene, clusterin. BJU Int 2009;103:384-90.

96. Gleave M, Miyake H. Use of antisense oligonucleotides targeting the cytoprotective gene, clusterin, to enhance androgen-and chemo-sensitivity in prostate cancer. World 
J Urol 2005;23:38-46.

97. Laskin JJ, Nicholas G, Lee C, et al. Phase I/II trial of custirsen (OGX-011), an inhibitor of clusterin, in combination with a gemcitabine and platinum regimen in patients with previously untreated advanced non-small cell lung cancer. J Thorac Oncol 2012;7:579-86.

98. Saad F, Hotte S, North S, et al. Randomized phase II trial of Custirsen (OGX-011) in combination with docetaxel or mitoxantrone as second-line therapy in patients with metastatic castrate-resistant prostate cancer progressing after first-line docetaxel: CUOG trial P-06c. Clin Cancer Res 2011;17:5765-73.

99. Chi KN, Higano CS, Blumenstein BA, et al. Phase III SYNERGY trial: Docetaxel+/-custirsen and overall survival in patients (pts) with metastatic castration-resistant prostate cancer (mCRPC) and poor prognosis. 2015.

100. García-Aranda M, Téllez T, Muñoz M, et al. Clusterin inhibition mediates sensitivity to chemotherapy and radiotherapy in human cancer. Anticancer Drugs 2017;28:702-16.

101. Gray LH, Conger AD, Ebert M, et al. The concentration of oxygen dissolved in tissues at the time of irradiation as a factor in radiotherapy. Br J Radiol 1953;26:638-48.

102.Zellweger T, Chi K, Miyake H, et al. Enhanced radiation sensitivity in prostate cancer by inhibition of the cell survival protein clusterin. Clin Cancer Res 2002;8:3276-84.

103. Yamanaka K, Gleave M, Muramaki M, et al. Enhanced radiosensitivity by inhibition of the anti-apoptotic gene clusterin using antisense oligodeoxynucleotide in a human bladder cancer model. Oncol Rep 2005;13:885-90.

104. Cao C, Shinohara ET, Li H, et al. Clusterin as a therapeutic target for radiation sensitization in a lung cancer model. Int J Radiat Oncol Biol Phys 2005;63:1228-36.

105. Watari H, Kinoshita R, Han Y, et al. Prognostic significance of clusterin expression in advanced-stage cervical cancer treated with curative intended radiotherapy. Int J Gynecol Cancer 2012;22:465-70.

106. Chen X, Bode AM, Dong Z, et al. The epithelialmesenchymal transition (EMT) is regulated by oncoviruses in cancer. FASEB J 2016;30:3001-10.

107. Yang S, Liu Y, Li MY, et al. FOXP3 promotes tumor growth and metastasis by activating $\mathrm{Wnt} / \beta$-catenin signaling pathway and EMT in non-small cell lung cancer. Mol Cancer 2017;16:124.

108.Lenferink AEG, Cantin C, Nantel A, et al. Transcriptome profiling of a TGF- $\beta$-induced epithelial-to-mesenchymal transition reveals extracellular clusterin as a target for therapeutic antibodies. Oncogene 2010;29:831-44.
109. Shiota M, Zoubeidi A, Kumano M, et al. Twist1 and clusterin regulate epithelial-mesenchymal transition, 2011.

110. Tian Y, Wang C, Chen S, et al. Extracellular Hsp90 $\alpha$ and clusterin synergistically promote breast cancer epithelialto-mesenchymal transition and metastasis via LRP1. J Cell Sci 2019;132:jcs228213.

111.Jin R, Chen X, Han D, et al. Clusterin modulates transdifferentiation of non-small-cell lung cancer. BMC Cancer 2017;17:661.

112. Li J, Yang S, Yan W, et al. Microrna-19 triggers epithelialmesenchymal transition of lung cancer cells accompanied by growth inhibition. Lab Invest 2015;95:1056-70.

113. Lin SH, Wang J, Saintigny P, et al. Genes suppressed by DNA methylation in non-small cell lung cancer reveal the epigenetics of epithelial-mesenchymal transition. BMC Genomics 2014;15:1079.

114. Barr S, Thomson S, Buck E, et al. Bypassing cellular EGF receptor dependence through epithelial-to-mesenchymallike transitions. Clin Exp Metastasis 2008;25:685-93.

115. Yauch RL, Januario T, Eberhard DA, et al. Epithelial versus mesenchymal phenotype determines in vitro sensitivity and predicts clinical activity of erlotinib in lung cancer patients. Clin Cancer Res 2005;11:8686-98.

116.He R, Hu Zhang F, Shen N. LncRNA FEZF1-AS1 enhances epithelial-mesenchymal transition (EMT) through suppressing E-cadherin and regulating WNT pathway in non-small cell lung cancer (NSCLC). Biomed Pharmacother 2017;95:331-8.

117.Petrova YI, Schecterson L, Gumbiner BM. Roles for E-cadherin cell surface regulation in cancer. Mol Biol Cell 2016;27:3233-44.

118. Chen D, Wang Y, Zhang K, et al. Antisense oligonucleotide against clusterin regulates human hepatocellular carcinoma invasion through transcriptional regulation of matrix metalloproteinase-2 and e-cadherin. Int J Mol Sci 2012;13:10594-607.

119. Tremblay GB, Viau E, Filion M. Co-use of a clusterin inhibitor with an egfr inhibitor to treat cancer: U.S. Patent 9,822,170. 2017.

120.Lombaerts M, Van Wezel T, Philippo K, et al. E-cadherin transcriptional downregulation by promoter methylation but not mutation is related to epithelial-to-mesenchymal transition in breast cancer cell lines. Br J Cancer 2006;94:661-71.

121. Theys J, Jutten B, Habets R, et al. E-Cadherin loss associated with EMT promotes radioresistance in human tumor cells. Radiother Oncol 2011;99:392-7.

122. Yoshino I, Kometani T, Shoji F, et al. Induction of 
epithelial-mesenchymal transition-related genes by benzo [a] pyrene in lung cancer cells. Cancer 2007;110:369-74.

123. Wu Y, Niu Y, Leng J, et al. Benzo(a)pyrene regulated A549 cell migration, invasion and epithelial-mesenchymal transition by up-regulating long non-coding RNA linc00673. Toxicology letters 2020;320:37-45.

124. Kim S, Koh J, Kim MY, et al. PD-L1 expression is associated with epithelial-to-mesenchymal transition in adenocarcinoma of the lung. Hum Pathol 2016;58:7-14.

125. Asgarova A, Asgarov K, Godet Y, et al. PD-L1 expression is regulated by both DNA methylation and NF- $\mathrm{kB}$ during EMT signaling in non-small cell lung carcinoma. Oncoimmunology 2018;7:e1423170.

126. Manjunath Y, Upparahalli SV, Avella DM, et al. PD-

Cite this article as: Tan J, Guo W, Yang S, Han D, Li H. The multiple roles and therapeutic potential of clusterin in nonsmall-cell lung cancer: a narrative review. Transl Lung Cancer Res 2021;10(6):2683-2697. doi: 10.21037/tlcr-20-1298
L1 expression with epithelial mesenchymal transition of circulating tumor cells is associated with poor survival in curatively resected non-small cell lung cancer. Cancers (Basel) 2019;11:806.

127. Raimondi C, Carpino G, Nicolazzo C, et al. PD-L1 and epithelial-mesenchymal transition in circulating tumor cells from non-small cell lung cancer patients: a molecular shield to evade immune system?. Oncoimmunology 2017;6:e1315488.

128. Chen L, Gibbons DL, Goswami S, et al. Metastasis is regulated via microRNA-200/ZEB1 axis control of tumour cell PD-L1 expression and intratumoral immunosuppression. Nat Commun 2014;5:5241. 